\title{
On the problem of linearizability of a 3-web
}

\author{
Zoltán Muzsnay
}

August 3, 2018

\begin{abstract}
In this paper we study the linearizability problem for 3-webs on a 2-dimensional manifold. With an explicit computation based on the theory developed in [9], we examine a 3 -web whose linearizability was claimed in 9 . We show that, contrary to the statement of [6] and [7, this particular web is linearizable. We compute explicitly the affine deformation tensor and the corresponding flat linear connection adapted to the web which linearizes the 3 -web.
\end{abstract}

AMS Classification: 53A60, 53C36

Keywords: Webs, linearization, affine structures, Gronwall conjecture.

\section{Introduction}

On a two-dimensional real or complex differentiable manifold $M$ a 3 -web is given by three foliations of smooth curves in general position. Two webs $\mathcal{W}$ and $\tilde{\mathcal{W}}$ are locally equivalent at $p \in M$, if there exists a local diffeomorphism on a neighborhood of $p$ which exchanges them. A 3-web is called linear (resp. parallel) if it is given by 3 foliations of straight lines (resp. of parallel lines). A 3-web which equivalent to a linear (resp. parallel) web is called linearizable (resp. parallelizable). An elegant characterization of parallelizable webs can be given in terms of the Chern connection associated to a 3-web: a 3-web is parallelizable if and only if the curvature of the Chern connection vanishes.

The problem of finding linearizability criterion is a very natural one. Bol suggested a method in 3] how to find a criterion of linearizability, although he was unable to carry out the computation. He showed that the number of projectively different linear 3 -webs in the plane which are equivalent to a non-parallelizable 3 -web is finite and less that 17 . The formulation of the linearizability problem in terms of the Chern connection was suggested by Akivis in a lecture given in Moscow in 1973. In his approach the linearizability problem is reduced to the solvability of a system of partial differential equations on the components of the affine deformation tensor. Using Akivis' idea Goldberg determined in [5] the first integrability conditions of the system.

By using this approach Grifone, Muzsnay and Saab solved the linearizability problem [9. They showed that, in the non-parallelizable case, there exists an algebraic submanifold $\mathcal{A}$ of the space of vector valued symmetric tensors $\left(S^{2} T^{*} \otimes T\right)$ on a neighborhood of $p$, expressed in terms of the curvature of the Chern connection and its covariant derivatives up to order 6 , so that the affine deformation tensor is a section of $S^{2} T^{*} \otimes T$ with values in $\mathcal{A}$. In particular: 
1. The web is linearizable if and only if $\mathcal{A} \neq \emptyset$;

2. There exists at most 15 projectively nonequivalent linearizations of a nonparallelizable 3-web.

The expressions of the polynomials and their coefficients which define $\mathcal{A}$ can be found in [10. The criteria of linearizability gives the possibility to make explicit computation on concrete examples to decide whether or not they are linearizable.

Recently Goldberg and Lychagin find similar results on the linearizability in [6], but their method is different from that of [9]. Despite of the fact, that the two theories concern the same problem and the final results are very similar, by testing them on an explicit example they lead to different answers. Indeed, considering the 3 -web $\mathcal{W}$ determined by the web function $f(x, y):=(x+y) e^{-x}$, i.e. the 3 -web given by the foliations

$$
x=\text { const }, \quad y=\text { const }, \quad(x+y) e^{-x}=\text { const. }
$$

we can find in [9] that $W$ is linearizable while [7] and 6] state the opposite.

In this present paper, with a computation based on the theory of [9], we prove that the 3 -web given by (1) is linearizable by finding explicitly the affine deformation tensor. Through this example we demonstrate the efficiency and the correctness of the approach developed in 9 .

\section{Basic notations and definitions}

Let $M$ be a 2-dimensional differentiable manifold.

Definition 2.1 $A 3$-web on $M$ is a triple of foliations $\left\{F_{1}, F_{2}, F_{3}\right\}$ such that the tangent spaces to the leaves of any two different foliations are complementary subspaces of $T$.

We will call the leaves of the foliations $\left\{F_{1}, F_{2}, F_{3}\right\}$ horizontal, vertical and transversal. Likewise, we call their tangent spaces horizontal, vertical and transversal and denote them by $T^{h}, T^{v}$ and $T^{t}$. We will use Nagy's formalism (cf. [12]). In particular, $h$ (resp. $v$ ) is the horizontal (resp. vertical) projection, $j$ is the associated product structure, $\nabla$ is the Chern connection. By the inverse functions theorem, we can find local coordinates $\left(x_{1}, x_{2}\right)$ at a neighborhood of $p \in M$ such that $\mathcal{W}$ can be written as

$$
x_{1}=\text { const }, \quad x_{2}=\text { const }, \quad f\left(x_{1}, x_{2}\right)=\text { const } .
$$

Using (2) as a local representation of the web, at every point the horizontal, vertical and transversal spaces are:

$$
T^{h}=\operatorname{Span}\left\{\partial_{1}\right\}, \quad T^{v}=\operatorname{Span}\left\{\partial_{2}\right\}, \quad T^{v}=\operatorname{Span}\left\{\partial_{1}-c\left(x_{1}, x_{2}\right) \partial_{2}\right\},
$$

where $c\left(x_{1}, x_{2}\right):=\partial_{1} f / \partial_{2} f$ and $\partial_{i}:=\partial / \partial x_{i}$. Moreover, one has $j\left(\partial_{1}\right)=c \partial_{2}$ and $j\left(\partial_{2}\right)=$ $\frac{1}{c} \partial_{1}$, therefore the basis

$$
\left\{e_{1}:=\partial_{1}, e_{2}:=c \partial_{2}\right\}
$$

is an adapted basis of the web i.e. $e_{1} \in T^{h}, e_{2} \in T^{v}$ and $j e_{1}=e_{2}$. The Chern connection $\nabla$ is determined by

$$
\nabla_{\partial_{1}} \partial_{1}=\Gamma_{1} \partial_{1}, \quad \nabla_{\partial_{2}} \partial_{2}=\Gamma_{2} \partial_{2}, \quad \nabla_{\partial_{1}} \partial_{2}=0, \quad \nabla_{\partial_{2}} \partial_{1}=0
$$


where $\Gamma_{1}=\frac{c_{1}}{c}$ and $\Gamma_{2}=-\frac{c_{2}}{c}$. The curvature tensor $R^{\nabla}$ of the Chern connection is characterized by the function

$$
r:=\frac{c_{1} c_{2}-c_{12} c}{c^{2}}=\frac{f_{11} f_{2}^{2} f_{12}-f_{1}^{2} f_{12} f_{22}-f_{1} f_{112} f_{2}^{2}+f_{1}^{2} f_{122} f_{2}}{f_{2}^{2} f_{1}^{2}},
$$

since for $i=1,2$ one has

$$
R^{\nabla}\left(\partial_{1}, \partial_{2}\right) \partial_{i}=r \partial_{i}
$$

\section{The PDE system of linearization}

Definition 3.1 A 3-web on a 2-dimensional affine space is called linear (resp. parallel) if the leaves of the three foliations are straight lines (resp. parallel straight lines). A 3-web on $M$ is called linearizable (resp. parallelizable) at $p \in M$ if it is equivalent to a linear (resp. parallel) 3-web modulo a local diffeomorphisms.

The problem of linearizability of webs can be formulated as follows: find a torsion-free flat connection $\nabla^{L}$ such that the foliations of the web are geodesic with respect to this connection [5]. The existence of such connection is equivalent to the existence of a symmetric (1,2)tensor field $L$, the linearization or affine deformation tensor, which satisfies to the condition that the connection $\nabla^{L}$ defined as

$$
\nabla_{X}^{L} Y:=\nabla_{X} Y+L(X, Y)
$$

preserves the web and flat. A tensor field $L$ in $S^{2} T^{*} \otimes T$ is a linearization if and only if

1. $v L(h X, h Y)=0$,

2. $h L(v X, v Y)=0$,

3. $L(h X, h Y)+j L(j h X, j h Y)-h L(j h X, h Y)$

$$
-h L(h X, j h Y)-j v L(j h X, h Y)-j v L(h X, j h Y)=0,
$$

4. $\nabla_{X} L(Y, Z)-\nabla_{Y} L(X, Z)+L(X, L(Y, Z))-L(Y, L(X, Z))+R^{\nabla}(X, Y) Z=0$,

holds for any $X, Y, Z \in T, 9$. Using local coordinate system, a symmetrical tensor $L=$ $L_{i j}^{k} d x^{i} \otimes d x^{j} \otimes \partial_{k} \in S^{2} T^{*} \otimes T$ is a linearization if and only if its components satisfy (4) and (5), where

$$
L_{11}^{2}=0, \quad L_{22}^{1}=0, \quad L_{12}^{2}=\frac{1}{2}\left(L_{11}^{1}+c L_{22}^{2}-2 c L_{12}^{1}\right),
$$

is a system of algebraic equations and

$$
\left.\begin{array}{r}
r+\frac{\partial L_{12}^{1}}{\partial x}-\frac{\partial L_{11}^{1}}{\partial y}+L_{12}^{2} L_{12}^{1}=0, \\
\frac{\partial L_{12}^{2}}{\partial x}-\Gamma_{1} L_{12}^{2}+L_{12}^{2} L_{12}^{2}-L_{11}^{1} L_{12}^{2}=0, \\
-\frac{\partial L_{12}^{1}}{\partial y}+\Gamma_{2} L_{12}^{1}+L_{22}^{2} L_{12}^{1}-L_{12}^{1} L_{12}^{1}=0, \\
r+\frac{\partial L_{22}^{2}}{\partial x}-\frac{\partial L_{12}^{2}}{\partial y}-L_{12}^{1} L_{12}^{2}=0 .
\end{array}\right\}
$$


is a system of first order quasi-linear partial differential equations.

A tensor $L$ in $S^{2} T^{*} \otimes T$ satisfying the algebraic conditions (4) called prelinearization. The prelinearizations forms a 3 dimensional subbundle of $S^{2} T^{*} \otimes T$ which will be denoted by $\mathcal{E}$. A section of $\mathcal{E}$ is a linearization if it satisfies (5).

\section{Sketch of the solution of the system of linearization}

In this section we describe the steps needed to solve the PDE system of linearization of the affine deformation tensor. It is composed of the equations of (4) and (5). The method used here is the same as the one used in [9] but here the system is written in terms of functions and and partial derivatives instead of tensors (see 9], p. 2648) and covariant derivatives.

As $\mathcal{E}$ is a rank-3 vector bundle, it can be parameterized by $\{s, t, z\}$, where

$$
s:=2 c L_{12}^{1}-c L_{22}^{2}, \quad t:=L_{12}^{2}, \quad z:=L_{12}^{1} .
$$

The parameter $s$ is called the base of the prelinearization and it is a projective invariant of the linearizations: two prelinearizations are projectively equivalent if and only if they have the same base [9. Writing the system (5) with $\{s, t, z\}$, the partial derivatives $t_{1}, t_{2}, z_{1}, z_{1}$ can be expressed explicitly as

$$
\begin{gathered}
t_{1}=t s+\frac{t\left(f_{11} f_{2}-f_{1} f_{12}+t f_{2} f_{1}\right)}{f_{2} f_{1}}, \\
t_{2}=t z+\frac{f_{2}^{3} f_{1} s_{1}-f_{2}^{3} f_{11} s+f_{12} f_{2}^{2} f_{1} s-2 f_{2}^{2} f_{1}^{2} s_{2}}{3 f_{2}^{2} f_{1}^{2}} \\
\quad+\frac{f_{11} f_{2}^{2} f_{12}-f_{1}^{2} f_{12} f_{22}+f_{1}^{2} f_{122} f_{2}-f_{1} f_{112} f_{2}^{2}}{3 f_{2}^{2} f_{1}^{2}}, \\
z_{1}=t z+\frac{-s_{2} f_{2}^{2} f_{1}^{2}+2 f_{2}^{3} f_{1} s_{1}-2 f_{2}^{3} f_{11} s+2 f_{12} f_{2}^{2} f_{1} s}{3 f_{2}^{2} f_{1}^{2}} \\
\quad+\frac{f_{1} f_{112} f_{2}^{2}-f_{11} f_{2}^{2} f_{12}+f_{1}^{2} f_{12} f_{22}-f_{1}^{2} f_{122} f_{2}}{3 f_{2}^{2} f_{1}^{2}}, \\
z_{2}=z^{2}-\frac{z\left(f_{12} f_{2}-f_{1} f_{22}+f_{2}^{2} s\right)}{f_{2} f_{1}} .
\end{gathered}
$$

By the consideration the integrability conditions $t_{12}=t_{21}$ and $z_{12}=z_{21}$ one can realize, that the functions $t, z$ and their derivatives can be eliminated. That way one obtains two second order PDE on $s$ :

$$
\begin{aligned}
\text { I.) } & 0=s_{11}-2 c s_{12}+\text { lower order terms... } \\
\text { II.) } & 0=s_{22}-\frac{2}{c} s_{12}+\text { lower order terms... }
\end{aligned}
$$

There is no integrability condition coming for the first prolongation of (17) and (8), but there is one integrability condition coming for the second prolongation. Indeed, using the second prolongation of (7) and (8), the equation

$$
0=c\left(\partial_{11} \mathrm{II}-\partial_{22} \mathrm{I}\right)+2\left(\partial_{12} \mathrm{I}-c^{2} \partial_{12} \mathrm{II}\right)
$$

does not contain 4 th order derivatives of $s$. We express the third order derivatives of $s$ form (7) and (8) and substitute them into (9), so we obtain a new equation:

$$
\text { III.) } \quad 0=24 \text { crs } s_{12}+\text { lower order terms... }
$$


We remark that the integrability condition (10) is identically satisfied if $r=0$, i.e. the web is parallelizable. If $r \neq 0$, the we have to push forward the computation.

Let us suppose that $r \neq 0$. We have to consider the system formed by (8), (17) and (10), which are second order PDE equations on $s$. The prolongation of these equations leads us to 2 integrability conditions. Indeed, considering the combinations

$$
\begin{aligned}
& 0=24 c r \mathrm{I}_{2}-\mathrm{III}_{1}+2 c \mathrm{III}_{2} \\
& 0=24 c r \mathrm{II}_{1}-\mathrm{III}_{2}+\frac{2}{c} \mathrm{III}_{1}
\end{aligned}
$$

the new equations do not contain 3rd order derivatives. Moreover the second order derivatives of $s$ can be expressed form (7), (8) and (9), we can substitute them into (11) and (12). That way we obtain two new equations having special forms

$$
\begin{aligned}
& 0=-24 r\left(s_{1}\right)^{2}+48 r s_{1} s_{2}+\alpha_{1} s_{1}+\beta_{1} s_{2}+\gamma_{1} \\
& 0=c 24 r\left(s_{2}\right)^{2}+48 r s_{1} s_{2}+\alpha_{2} s_{1}+\beta_{2} s_{2}+\gamma_{2}
\end{aligned}
$$

where $\alpha^{i}, \beta^{i}$ and $\gamma^{i}(i=1,2)$ are determined by the curvature and its derivatives. At the final step one has to take the derivatives of (13) and (14) with respect to the variables $x_{1}$ and $x_{2}$. By expressing $\left(s_{1}\right)^{2}$ and $\left(s_{2}\right)^{2}$ from (13) and (14), respectively, and by putting the correspondent values into the prolongated derived system we obtain a system of for equations

$$
a^{i} s_{1}+b^{i} s_{2}+c^{i} s_{1} s_{2}=d^{i}, \quad i=1, . ., 4 .
$$

where $a^{i}, b^{i}, c^{i}$ and $d^{i}, i=1, . ., 4$ are determined by the curvature and its derivatives. (15) can be considered as a linear system in $s_{1}, s_{2}$ and $s_{1} s_{2}$. This system is compatible, and the $3^{\text {rd }}$-order minors are non zero polynomials in $s$ of degree 7 (9], p. 2652). So there exists an open $\mathcal{U} \subset \mathbb{C}^{2}$ on which

$$
D(s):=\left|\begin{array}{lll}
a^{1} & b^{1} & c^{1} \\
a^{2} & b^{2} & c^{2} \\
a^{3} & b^{3} & c^{3}
\end{array}\right| \neq 0 .
$$

Solving on $\mathcal{U}$ the linear system (15) by the Cramer formulas, we get:

$$
s_{1}=\frac{A(s)}{D(s)}, \quad s_{2}=\frac{B(s)}{D(s)}, \quad s_{1} s_{2}=\frac{C(s)}{D(s)},
$$

where $A, B$, and $C$ are given by the corresponding determinant. These functions are polynomial in $s$. Moreover,

(a) using the identity $s_{1} \cdot s_{2}=s_{1} s_{2}$ and the expression of the corresponding terms given by (16) we obtain that the solution $s$ of the linearization system has to take his values on algebraic manifold defined by $Q_{1}(s)=0$, where $Q_{1}(s):=A B-C D$ is polynomial in $s$ of degree 18.

(b) For the system (16) the compatibility condition is given by $\partial_{1} s_{2}-\partial_{2} s_{1}=0$. Using $A$, $B$ and $D$, we obtain that $s$ has to take its values in the algebraic manifold $Q_{2}(s)=0$ defined by this compatibility condition.

(c) Just like the first derivatives are computed in (16), the second order derivatives can also be expressed in a similar way, and using their expressions in the equations (7), (8), (10), (13) and (14) we get 5 polynomial equations in $s: Q_{i}=0,(i=3, \ldots, 7)$.

It follows that $s=s\left(x_{1}, x_{2}\right)$ has to take its values in the algebraic manifold $\mathcal{A} \subset E$, where

$$
\mathcal{A}:=\left\{Q_{i}=0 \mid i=1, \ldots, 7\right\} .
$$




\section{Example}

In this section we consider the 3 -web determined by the web function $f\left(x_{1}, x_{2}\right):=\left(x_{1}+\right.$ $\left.x_{2}\right) e^{-x_{1}}$, that is the 3 -web $\mathcal{W}$ given by the foliations

$$
x_{1}=\text { const }, \quad x_{2}=\text { const }, \quad\left(x_{1}+x_{2}\right) e^{-x_{1}}=\text { const } .
$$

The linearizability of this example was examined by Grifone, Muzsnay and Saab in 9] (page 2563), and the authors claimed that this particular web is linearizable. However, in 6] (page 38) and [7 (page 171) the authors stated the opposite.

Let us examine this example more closely. The Chern connection of the web $\mathcal{W}$ is determined by:

$$
\begin{aligned}
\nabla_{\partial_{1}} \partial_{1} & =\frac{1}{x_{1}+x_{2}-1} \partial_{1}, & \nabla_{\partial_{1}} \partial_{2} & =0, \\
\nabla_{\partial_{2}} \partial_{2} & =\frac{1}{1-x_{1}-x_{2}} \partial_{2}, & \nabla_{\partial_{2}} \partial_{1} & =0 .
\end{aligned}
$$

The curvature is given by

$$
R^{\nabla}\left(\partial_{1}, \partial_{2}\right) \partial_{i}=\frac{1}{\left(x_{1}+x_{2}-1\right)^{2}} \partial_{i}
$$

for $i=1,2$. Therefore the Chern connection is non flat and the web $\mathcal{W}$ is not parallelizable. Following the computation described in the previous chapter one can find, that

$$
s\left(x_{1}, x_{2}\right) \equiv-1
$$

is a solution for all the polynomials $Q_{i}(s), i=1, \ldots, 7$. This shows that the web is linearizable. Let us go further and find the linearization explicitly. By substituting $s\left(x_{1}, x_{2}\right) \equiv-1$ into (6) one obtains that

$$
\begin{aligned}
& t_{1}=t^{2}-t+\frac{t}{x_{1}+x_{2}-1}, \\
& t_{2}=t z \\
& z_{1}=t z-\frac{1}{\left(x_{1}+x_{2}-1\right)^{2}}, \\
& z_{2}=z^{2}-\frac{2 z}{x_{1}+x_{2}-1} .
\end{aligned}
$$

There are two solutions of the differential system (19):

$$
\begin{aligned}
& \text { Solution 1. }\left\{\begin{array}{l}
t\left(x_{1}, x_{2}\right)=0, \\
z\left(x_{1}, x_{2}\right)=\frac{1-x_{1}-a}{\left(-1+x_{1}+x_{2}\right)\left(x_{2}-a\right)},
\end{array}\right. \\
& \text { Solution 2. }\left\{\begin{array}{l}
t\left(x_{1}, x_{2}\right)=\frac{\left(-1+x_{1}+x_{2}\right) e^{-x_{1}}}{\left(x_{1}+x_{2}\right) e^{-x_{1}}+a x_{2}+b}, \\
z\left(x_{1}, x_{2}\right)=\frac{e^{-x_{1}}+a-x_{1} a+b}{\left(\left(x_{1}+x_{2}\right) e^{-x_{1}}+a x_{2}+b\right)\left(x_{1}+x_{2}-1\right)}
\end{array}\right.
\end{aligned}
$$


where $a$ and $b$ are arbitrary constants.

Solution 1. Here we consider the solution (20) of (19). Rewriting the expression of $t\left(x_{1}, x_{2}\right)$ and $z\left(x_{1}, x_{2}\right)$ with the help of (18) we can determine the components of the affine deformation tensor $L$ :

$$
\begin{array}{ll}
L_{11}^{1}=-1, & L_{22}^{2}=-\frac{x_{2}-2+2 x_{1}+a}{\left(x_{1}+x_{2}-1\right)\left(x_{2}-a\right)}, \\
L_{12}^{2}=0, & L_{12}^{1}=\frac{1-x_{1}-a}{\left(-1+x_{1}+x_{2}\right)\left(x_{2}-a\right)} .
\end{array}
$$

The deformed connection $\nabla^{L}$ in the standard base is given by the following equations:

$$
\begin{aligned}
& \nabla_{\partial_{1}}^{L} \partial_{1}=\nabla_{\partial_{1}} \partial_{1}+L\left(\partial_{1}, \partial_{1}\right)=\frac{c_{1}}{c} \partial_{1}+L_{11}^{1} \partial_{1}=\frac{x_{1}+x_{2}-2}{1-x_{1}-x_{2}} \partial_{1} \\
& \nabla_{\partial_{1}}^{L} \partial_{2}=\nabla_{\partial_{1}} \partial_{2}+L\left(\partial_{1}, \partial_{2}\right)=L_{12}^{1} \partial_{1}+L_{12}^{2} \partial_{2}=\frac{1-x_{1}-a}{\left(-1+x_{1}+x_{2}\right)\left(x_{2}-a\right)} \partial_{1} \\
& \nabla_{\partial_{2}}^{L} \partial_{1}=\nabla_{\partial_{2}} \partial_{1}+L\left(\partial_{2}, \partial_{1}\right)=L_{12}^{1} \partial_{1}+L_{12}^{2} \partial_{2}=\frac{1-x_{1}-a}{\left(-1+x_{1}+x_{2}\right)\left(x_{2}-a\right)} \partial_{1} \\
& \nabla_{\partial_{2}}^{L} \partial_{2}=\nabla_{\partial_{2}} \partial_{2}+L\left(\partial_{2}, \partial_{2}\right)=-\frac{c_{2}}{c} \partial_{2}+L_{22}^{2} \partial_{2}=\frac{2}{a-x_{2}} \partial_{2}
\end{aligned}
$$

It is obvious that $\nabla_{\partial_{i}}^{L} \partial_{j}-\nabla_{\partial_{j}}^{L} \partial_{i}=0$ and therefore the torsion of $\nabla^{L}$ is zero. Moreover, at every point the horizontal, vertical and transversal spaces are:

$$
T^{h}=\operatorname{Span}\left\{\partial_{1}\right\}, \quad T^{v}=\operatorname{Span}\left\{\partial_{2}\right\}, \quad T^{v}=\operatorname{Span}\left\{\partial_{1}-c \partial_{2}\right\},
$$

where $c=\partial_{1} f / \partial_{2} f=1-x_{2}-x_{2}$. The equation (22) (resp. (25)) shows that the covariant derivative of a horizontal (resp. vertical) vectorfield with respect to a horizontal (resp. vertical) vectorfield is horizontal (resp. vertical). Moreover, we have

$$
\begin{aligned}
\nabla_{\left(\partial_{1}-c \partial_{2}\right)}\left(\partial_{1}-c \partial_{2}\right) & =\nabla_{\partial_{1}} \partial_{1}-c \nabla_{\partial_{2}} \partial_{1}-c \nabla_{\partial_{1}} \partial_{2}-\left(\partial_{1} c\right) \partial_{2}+c\left(\partial_{2} c\right) \partial_{2}+c^{2} \nabla_{\partial_{2}} \partial_{2} \\
& =\frac{2 x_{1}^{2}+x_{2}^{2}+3 x_{1} x_{2}+(a-4)\left(x_{1}+x_{2}\right)+2}{\left(-1+x_{1}+x_{2}\right)\left(a-x_{2}\right)}\left(\partial_{1}-c \partial_{2}\right)
\end{aligned}
$$

which shows that the covariant derivative of a transversal vectorfield with respect to a transversal vectorfield is transversal. Direct calculation shows that $\nabla^{L}$ is flat, that is its curvature tensor is identically zero.

Solution 2. Here we consider the solution (21) of (19). Completing the expression of $t\left(x_{1}, x_{2}\right)$ and $z\left(x_{1}, x_{2}\right)$ with (18) we can find that the components of $L$, the affine deformation tensor are:

$$
\begin{aligned}
L_{11}^{1} & =\frac{\left(x_{1}+x_{2}-2\right) e^{-x_{1}}-a x_{2}-b}{\left(x_{1}+x_{2}\right) e^{-x_{1}}+a x_{2}+b} \\
L_{22}^{2} & =\frac{\left(2-x_{1}-x_{2}\right) e^{-x_{1}}-a\left(2 x_{1}+x_{2}-2\right)+b}{\left(x_{1}+x_{2}-1\right)\left(\left(x_{1}+x_{2}\right) e^{-x_{1}}+a x_{2}+b\right)} \\
L_{12}^{1} & =\frac{e^{-x_{1}}-a x_{1}+a+b}{\left(x_{1}+x_{2}-1\right)\left(\left(x_{1}+x_{2}\right) e^{-x_{1}}+a x_{2}+b\right)} \\
L_{12}^{2} & =\frac{\left(x_{1}+x_{2}-1\right) e^{-x_{1}}}{\left(x_{1}+x_{2}\right) e^{-x_{1}}+a x_{2}+b}
\end{aligned}
$$


The deformed connection $\nabla^{L}$ in the standard base is given by the following equations:

$$
\begin{aligned}
& \nabla_{\partial_{1}}^{L} \partial_{1}=\left(\frac{1}{x_{1}+x_{2}-1}+\frac{\left(x_{1}+x_{2}-2\right) e^{-x_{1}}-a x_{2}-b}{\left(x_{1}+x_{2}\right) e^{-x_{1}}+a x_{2}+b}\right) \partial_{1} \\
& \nabla_{\partial_{1}}^{L} \partial_{2}=\frac{e^{-x_{1}}+a-x_{1} a+b}{\left(\left(x_{1}+x_{2}\right) e^{-x_{1}}+a x_{2}+b\right)\left(x_{1}+x_{2}-1\right)} \partial_{1}+\frac{\left(x_{1}+x_{2}-1\right) e^{-x_{1}}}{\left(x_{1}+x_{2}\right) e^{-x_{1}}+a x_{2}+b} \partial_{2}, \\
& \nabla_{\partial_{2}}^{L} \partial_{1}=\frac{e^{-x_{1}}+a-x_{1} a+b}{\left(\left(x_{1}+x_{2}\right) e^{-x_{1}}+a x_{2}+b\right)\left(x_{1}+x_{2}-1\right)} \partial_{1}+\frac{\left(x_{1}+x_{2}-1\right) e^{-x_{1}}}{\left(x_{1}+x_{2}\right) e^{-x_{1}}+a x_{2}+b} \partial_{2}, \\
& \nabla_{\partial_{2}}^{L} \partial_{2}=\frac{-2\left(e^{-x_{1}}+a\right)}{\left(x_{1}+x_{2}\right) e^{-x_{1}}+a x_{2}+b} \partial_{2}
\end{aligned}
$$

As in the previous case $\nabla_{\partial_{i}}^{L} \partial_{j}-\nabla_{\partial_{j}}^{L} \partial_{i}=0$ and the torsion of $\nabla^{L}$ is zero. Equation (26) (resp. (29) ) shows that the covariant derivative of a horizontal (resp. vertical) vectorfield with respect to a horizontal (resp. vertical) vectorfield is horizontal (resp. vertical). We have

$$
\begin{aligned}
& \nabla_{\left(\partial_{1}-c \partial_{2}\right)}\left(\partial_{1}-c \partial_{2}\right)= \\
& \quad=\frac{\left(x_{1}+x_{2}\right)^{2} e^{-x_{1}}+(4 a+b)\left(x_{1}+x_{2}\right)-a\left(2 x_{1}^{2}+x_{2}^{2}+3 x_{2} x_{1}+2\right)}{\left(x_{1}+x_{2}-1\right)\left(\left(x_{1}+x_{2}\right) e^{-x_{1}}+a x_{2}+b\right)}\left(\partial_{1}-c \partial_{2}\right)
\end{aligned}
$$

which shows that the covariant derivative of a transversal vectorfield with respect to a transversal vectorfield is transversal. As in the previous case, $\nabla^{L}$ is flat i.e. its curvature tensor is identically zero.

As the direct calculations show in both cases

1. the connection $\nabla^{L}$ preserves the web, that is the three families of leaves are autoparallels curves with respect to it;

2. $\nabla^{L}$ is torsion free;

3. $\nabla^{L}$ is flat, that is its curvature tensor is identically zero.

The properties 1.) - 3.) show that the corresponding affine deformation tensor $L$ in both cases is a linearization of the web $\mathcal{W}$.

Remark. Solution 1. and Solution 2. correspond to different linearizations. However, these linearizations are projectively equivalent. Indeed, the parameter $s$, called the base of the linearization, is a projective invariant of the linearizations: two linearizations are projectively equivalent if and only if they have the same base. Here the two linearizations have the same base $\left(s\left(x_{1}, x_{2}\right) \equiv-1\right)$ which shows that they are projectively equivalent.

\section{References}

[1] Akivis, M.A. And Shelkhov, A.M.; Geometry of Algebra of Multidimensional ThreeWebs. Kulwer Academic Publishers, Dordrecht (1992).

[2] Blaschke, W.; Einführung in die Geometrie der waben. Birkhauser-Verlag, BaselStuttgart, 1955. 
[3] Bol, G.; Geradlinige Kurvengewebe. Abh. Math. Sem. Univ. Hambourg, 8, (1930), 264-270.

[4] BoL, G.; Über Geradengewebe. Ann. Mat. Pura e Appl. (4) 17 (1938), 45-58.

[5] Goldberg, V.V.; On a linearizability condition for a three-web on a two-dimensional manifold. Differential Geometry, (Peniscola, 1988), 223-239, Lecture Notes in Math. 1410, Springer-Verlag, Berlin New-York, (1989).

[6] Goldberg,V.V.; Lychagin, V.V.; On the Blaschke conjecture for 3-webs, arXiv: math.DG/0411460

[7] Goldberg,V.V.; Lychagin, V.V.; On linearization of planar three-webs and Blaschke's conjecture, C.R.Acad. Sci. Paris, Ser. I. vol. 341. num 3 (2005)

[8] Grifone, J; Muzsnay, Z.; Variational Principles For Second-Order Differential Equations, World Scientific, Singapore, (2000).

[9] Grifone, J; Muzsnay, Z; SaAb J.; On the linearizability of 3-webs, Nonlinear analysis $47,(2001) 2643-2654$.

[10] Grifone, J; Muzsnay, Z; SaAb J.; Linearizable 3-webs and the Gronwall conjecture, arXiv: math.DG/0602535

[11] Gronwall, T.H. Sur les équations entre trois variables représentables par des nomogrammes à points alignés. J. de Liouville, 8, 59-102.

[12] NaGy, P.T.; Invariant tensorfields and the canonical connection of a 3-web. Aequationes Math, 35 (1988) 31-44.

Zoltán Muzsnay : University of Debrecen, Department of Mathematics, Debrecen, H-4032 PBox 12, Hungary,

E-mail address: muzsnay@math.klte.hu 